\title{
Plus n'est pas toujours plus. Décidons ensemble
}

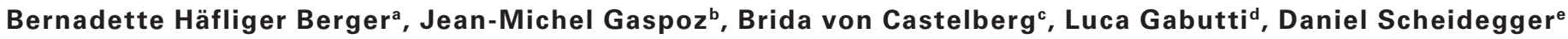 \\ a Avocate, directrice smarter medicine, secrétaire générale de la SSMIG; b Professeur, président smarter medicine, co-président de la SSMIG; \\ 'Docteure, membre du comité de smarter medicine, vice-présidente de I'OSP; d Professeur, membre du comité de smarter medicine; \\ e Professeur ém., membre du comité de smarter medicine, président de l'ASSM
}

L'association smarter medicine - Choosing Wisely Switzerland a lancé une campagne qui vise à mieux faire comprendre au grand public le thème de la surmédicalisation et des soins inappropriés. Les listes des traitements superflus des sociétés de discipline médicale ont été réécrites de façon à être accessibles aux personnes n’ayant pas de connaissances médicales. Le dialogue entre les personnes soignées et celles qui soignent est toutefois au cœur des préoccupations.

Le postulat du philosophe et médecin grec Epictète, posé il y a près de 2000 ans, est toujours d'actualité. «Les malades se fâchent contre les médecins qui ne leur prescrivent rien. Ils ont l'impression qu'ils les abandonnent.» Dans le système de santé moderne, on observe aussi une certaine crainte qu'il faille rationner les prestations médicales au détriment de certaines personnes. Compte tenu des progrès fulgurants de la médecine précisément, beaucoup sont convaincus qu'il faudrait systématiquement épuiser tous les moyens possibles. On se demande beaucoup trop rarement s'ils sont indiqués dans le cas spécifique et s'ils contribuent effectivement à la guérison des patients ou à l'amélioration de leur qualité de vie.

L'initiative smarter medicine adhère au principe selon lequel il faut renoncer à un traitement ou à une méthode de diagnostic dès l'instant où ils n'apportent aucun bénéfice aux patients, voire leur portent préjudice. L'association faîtière a été fondée l'an dernier et réunit des sociétés de discipline médicale et des organisations professionnelles, mais aussi des organisations de patients et de consommateurs.

\section{Seuls des patients bien informés et ayant}

confiance en eux sont en mesure de comprendre les décisions et de les assumer conjointement.

smarter medicine lance aujourd'hui une campagne de deux ans qui place le point de vue des consommateurs et des patients au cœur du débat, avec comme slogan «Plus n'est pas toujours plus. Décidons ensemble». Les patients doivent pouvoir discuter d'égal à égal avec ce- lui qui les soigne de la méthode de diagnostic et thérapeutique appropriée. Seuls des patients bien informés et ayant confiance en eux sont en mesure de comprendre les décisions et de les assumer conjointement. Ils doivent aussi pouvoir avoir la certitude de bénéficier chaque fois du meilleur traitement dans leur cas précis. Cela ne veut pas dire pour autant que tous les moyens disponibles doivent être mis en œuvre.

\section{La surmédicalisation et les soins inappro- priés sont-ils vraiment un problème?}

Les études montrent que les traitements non indiqués médicalement sont à l'origine de 20 à 30\% des dépenses de santé [1]. Ainsi, le nombre d'interventions en chirurgie articulaire enregistre une augmentation nettement supérieure à ce qui serait explicable d'un point de vue épidémiologique du fait du vieillissement de la population [2]. Une autre étude révèle que dans certains hôpitaux de Suisse, jusqu'à 70\% des cathétérismes cardiaques électifs sont réalisés sans le moindre résultat d'analyse attestant d'une pathologie [3]. smarter medicine a la conviction qu'il existe aujourd'hui une tendance dans le système de santé suisse à rendre les patients plus malades qu'ils ne le sont réellement [4]. L'une des raisons de cette surmédicalisation réside dans la haute densité de médecins spécialistes et dans le développement des sous-spécialisations. La fragmentation et le manque de coordination des soins médicaux pourraient constituer une autre cause. Le système tarifaire suisse contribue aussi à la surmédicalisation, étant donné que les examens 


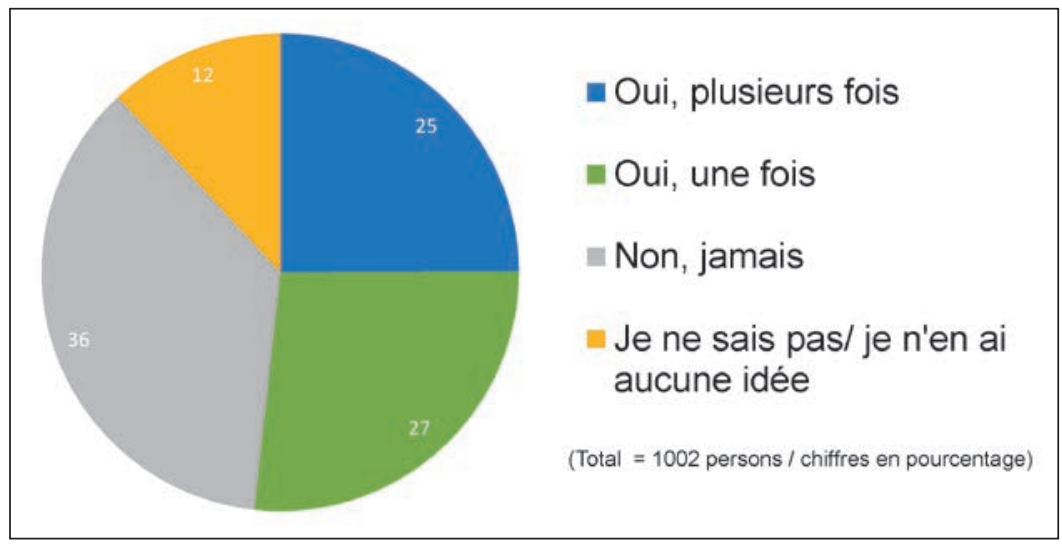

Fig. 1: GfK 2018 GfK eBus 2018 à propos de la question: Avez-vous ou une personne de votre entourage a-t-elle déjà eu le sentiment d'avoir reçu un traitement qui n'était pas nécessaire?

et traitements complémentaires permettent de gagner de l'argent.

Une campagne lancée en 2014 par le réseau des hôpitaux publics tessinois (Ente Ospedaliero Cantonale) montre qu'il est possible de corriger le tir. Le point de départ de cette intervention interprofessionnelle était le constat d'une prescription anormalement élevée de benzodiazépine et d'antiacide (inhibiteur de la pompe à protons), avec une variabilité pertinente entre les divers hôpitaux. L'intervention reposait initialement sur un monitoring et une comparaison avec des valeurs de référence. Le personnel impliqué a ensuite été directement contacté puis formé dans le cadre de séances d'information et de cours de formation continue. Cela a permis de réduire efficacement la prescription à long terme de ces deux médicaments $[5,6]$.

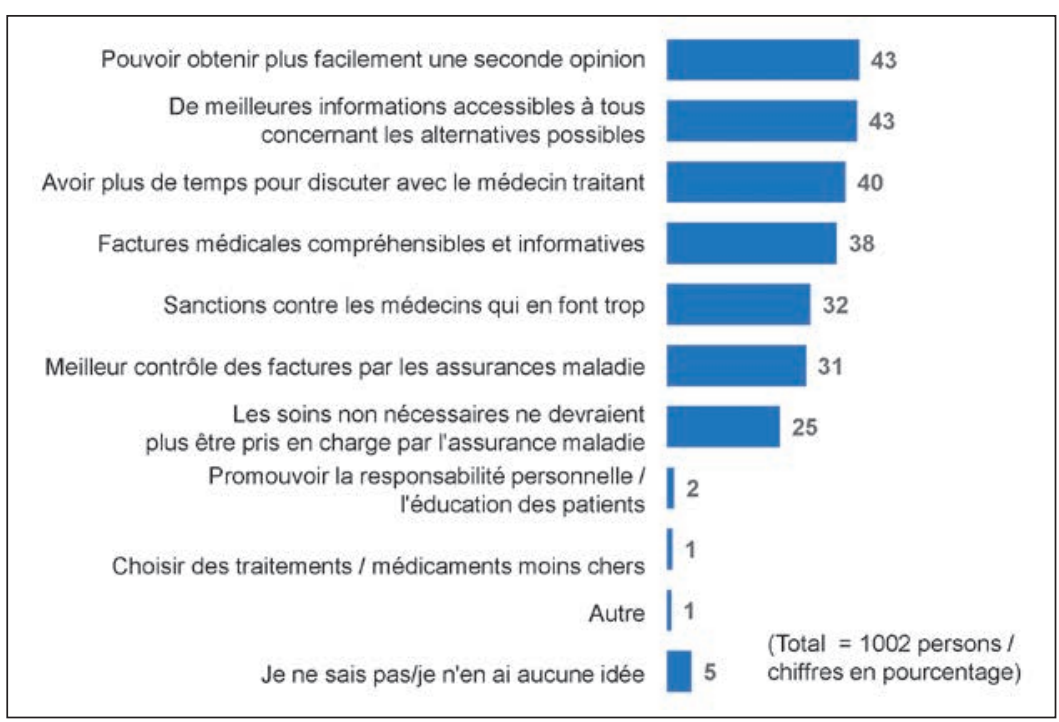

Fig. 2: GfK 2018 GfK eBus 2018 à propos de la question: Qu'est-ce qui contribuerait à éviter la surmédicalisation? Par "surmédicalisation», nous entendons la prescription de traitements superflus ou erronés.

\section{Que pensent les patients?}

Durant la période du 28 juin au 3 juillet 2018, l'institut de sondage d'opinion GfK a réalisé une enquête sur les soins médicaux et la notoriété de smarter medicine pour le compte de l'association faîtière. L'institut a reçu plus d'un millier de réponses en provenance de Suisse alémanique et romande et les résultats peuvent donc être considérés comme représentatifs. Cette enquête devra être renouvelée à la fin de la campagne afin de mesurer l'efficacité des mesures prises. Comme il fallait s'y attendre, peu de gens connaissent actuellement l'initiative (à peine 6\%).

Le problème abordé, celui de la surmédicalisation, est néanmoins connu. Plus d'une personne interrogée sur deux estime qu'elle-même ou quelqu'un de son entourage a déjà reçu - une fois ou à plusieurs reprises - un traitement qui n'aurait pas été nécessaire. A l'inverse, $36 \%$ des personnes interrogées ont la conviction qu'une telle chose ne leur est jamais arrivée (fig. 1).

A la question "Qu'est-ce qui contribuerait à éviter la surmédicalisation?», les personnes interrogées ont mentionné comme mesures principales l'accès plus aisé à un deuxième avis médical, des informations facilement accessibles sur les alternatives possibles et du temps supplémentaire pour discuter avec le médecin traitant. Toutefois, seules $2 \%$ des personnes interrogées estiment que la responsabilisation accrue des patients serait un outil approprié pour lutter contre la surmédicalisation. En revanche, plus de 30\% des personnes interrogées souhaitent que des sanctions soient prises à l'encontre des médecins qui en font trop (fig. 2).

Les résultats complets de l'enquête ont été publiés sur le site www.smartermedicine.ch.

\section{Les choses bougent au sein des sociétés de discipline médicale}

Les sociétés de discipline médicale jouent un rôle central dans l'initiative smarter medicine. Ce sont elles en effet qui élaborent et publient des recommandations les listes dites «Top 5»-pour leurs disciplines respectives. La liste Top 5 énumère cinq interventions à éviter. Ce sont donc les sociétés de discipline médicale qui fournissent le matériel de base en tant que tel. Lors de la fondation de l'association smarter medicine durant l'été 2017, toutes les sociétés de discipline médicale ont été invitées à élaborer et à publier des listes pour leur domaine. Dans l'intervalle, sept d'entre elles ont donné suite à cette demande. Et d'autres sont en train de rédiger de telles listes. L'objectif est toutefois que toutes les sociétés de discipline médicale ainsi que d'autres orga- 
nisations non médicales se joignent à cette initiative. Récemment, divers hôpitaux des cantons de Genève, de Zurich et du Tessin ont ainsi créé la plateforme «Smarter Hospital» [7].

\section{Des informations spécifiques et une première «Journée des patients»}

Entre-temps, les recommandations existantes ont été traduites en un langage plus simple et compréhensible. Cela permet aujourd'hui de mieux impliquer les patients. Les informations sont en priorité publiées sur le site Internet www.smartermedicine.ch et ainsi rendues accessibles au plus grand nombre. En parallèle, des vidéos explicatives sont utilisées et une campagne est réalisée sur les réseaux sociaux pour chercher à sensibiliser le public au thème de la surmédicalisation et des soins inappropriés. Le 20 septembre 2019, l'association devrait organiser une première Journée nationale des patients en collaboration avec différentes organisations du domaine de la santé.

\section{smarter medicine dénonce aussi le gaspillage des ressources dans le secteur de la santé.}

Correspondance: smarter medicineChoosing Wisely Switzerland c/o SSMIG Monbijoustrasse 43 Case postale CH-3001 Berne smartermedicine[at] sgaim.ch
Outre les informations spécifiquement destinées aux patients, à la demande du corps médical et en collaboration avec le Swiss Medical Board, les principales recommandations des sociétés de discipline médicale sont également complétées par des supports d'information supplémentaires (séries de fiches, affiches, etc.) pour les fournisseurs de prestations.

\section{Une stratégie également destinée à lutter contre le gaspillage}

smarter medicine dénonce aussi le gaspillage des ressources dans le secteur de la santé, car il entraîne une hausse des coûts tout en accentuant la pression qui pousse vers la rationalisation en médecine. Par sa campagne auprès des patients, l'association ne cherche pas a priori réaliser des économies, mais surtout à améliorer la qualité des soins médicaux. Il y a toutefois lieu de supposer que le respect systématique des recommandations contribuera aussi à réduire les dépenses de santé.

\section{Références}

1 Gerber M, Kraft E, Bosshard C. Overuse - unnötige Behandlungen als Qualitätsproblem. Schweiz Ärzteztg. 2016;97(7):236-43.

2 Rosemann T, Neuner-Jehle S, Muheim L. Versorgungsforschung: das richtige Mass an Medizin, zum richtigen Zeitpunkt für den richtigen Patienten. Therapeutische Umschau. 2017;74(1):827-31.

3 Interventional Cardiology in Switzerland - Annual Statistic Report. 2015. Swiss Working Group of Interventional Cardiology. http://www.ptca.ch/DOCS_PUBLIC/ptca_statistics_2015.pdf.

4 Anderegg S. «Es besteht der Anreiz, die Patienten kränker zu machen, als sie sind.» Tages-Anzeiger du 6 juin 2018.

5 Del Giorno R, Ceschi A, Pironi M, Zasa A, Greco A, Gabutti L. Multifaceted intervention to curb in-hospital over-prescription of proton pump inhibitors: A longitudinal multicenter quasi-experimental before-and-after study. Eur J Intern Med. 2018;50:52-9. Doi: 10.1016/j.ejim.2017.11.002.

6 Del Giorno R, Greco A, Zasa A, Clivio L, Pironi M, Ceschi A, Gabutti L. Combining prescription monitoring, benchmarking, and educational interventions to reduce benzodiazepine prescriptions among internal medicine inpatients; a multicenter before and after study in a network of Swiss Public Hospitals. Postgrad Med. 2018;2:1-10. Doi: 10.1080/00325481.2018.1504594.

7 Gaspoz J-M. Smarter Hospital - für eine Medizin mit Augenmass. PHC. 2018;18(14):241.

Crédits photo

Figures 1 et 2: ๑ initiative smarter medicine 